\title{
HYDRODYNAMICS OF RELATIVISTIC PULSAR WIND
}

\author{
J. G. LOMINADZE \\ Abastumani Astrophysical Observatory \\ J. I. JAVAKHISHVILI \\ Tbilisi State University \\ E. G. TSIKARISHVILI \\ Abastumani Astrophysical Observatory
}

Pulsar pair-production theories imply that the plasma leaves the source already flowing relativistically. Such winds might also have relativistic injection temperatures. MHD models of relativistic pulsar winds were considered, and it was shown that in this case cold wind theories can be misleading.

On the other hand, due to synchrotron radiation losses, the temperature of collisionless, strongly magnetized, relativistic plasma quickly becomes anisotropic. In this case the plasma pressure is no longer a scalar and to describe the properties of stellar wind a hydrodynamical model of relativistic plasma with the anisotropic temperature distribution should be developed.

In this paper we get a closed set of relativistic hydrodynamical equations, which describes relativistic strongly magnetized, collisionless plasma with an anisotropic pressure tensor. Such a model was suggested by Chew, Goldberger and Low (1954) for a nonrelativistic plasma.

The microscopic state of the relativistic collisionless plasma can be described by means of the plasma particle's relativistic distribution function $\Phi_{a}\left(x_{a}^{i}, p_{a}^{i}\right)$, which satisfies the kinetic equations

$$
\left[H \Phi_{a}\right]=\frac{\partial H}{\partial p_{a i}} \frac{\partial \Phi_{a}}{\partial x_{a}^{i}}-\frac{\partial H}{\partial x_{a i}} \frac{\partial \Phi_{a}}{\partial p_{a}^{i}}=0
$$

here $H$ is the Hamiltonian of the particle system, $a$ denotes the particle species, $x_{a}^{i}=\left(c t, \boldsymbol{r}_{a}\right)$ denotes a 4-space vector, $\boldsymbol{p}_{a}^{i}=\left(\varepsilon_{a} / c, \boldsymbol{p}_{a}\right)$ denotes the particle 4-momentum $\left(\varepsilon_{a}=c \sqrt{p_{a}^{2}+m_{a}^{2} c^{2}}\right), i=0,1,2,3$.

Of course, the following system of Hamilton equations is valid

$$
\frac{\partial H}{\partial p_{a i}}=\frac{d x_{a}^{i}}{d s_{a}}=\frac{p_{a}^{i}}{m_{a} c}, \quad \frac{\partial H}{\partial x_{a i}}=-\frac{d p_{a}^{i}}{d s_{a}}
$$

where $d s_{a}=\sqrt{d x_{a}^{i} d x_{a i}}=c \gamma_{a}^{-1} d t$, and $\gamma_{a}=1 / \sqrt{1-u_{a}^{2} / c^{2}}$ is the relativistic factor.

Let us consider the plasma in the presence of electric and magnetic fields. The particle equation of motion is as follows (Landau and Lifshitz 1967)

$$
\frac{d p_{a}^{i}}{d s_{a}}=\frac{e_{a}}{c} F^{i j} \frac{p_{a j}}{m_{a} c}
$$

Using equations (2) and (3) one can reduce eq.(1) to the following form

$$
p_{a}^{i} \frac{\partial \Phi_{a}}{\partial x_{a}^{i}}+\frac{e_{a}}{c} F^{i j} p_{a j} \frac{\partial \Phi_{a}}{\partial p_{a i}}=0
$$

It is well known (Baranov and Krasnobaev 1986), that from the kinetic equations one can obtain the system of transport equations for the macroscopic plasma parameters (e.g. for the particle density $n_{a}$, hydrodynamical velocity $u_{a}$, and for the mean thermal energy $m_{a} c^{2} W_{a}$-taken in the particle rest frame). Hence for each plasma component the following definitions are introduced (Landau and Lifshitz 1986)

$$
I_{a}^{i}=\int p_{a}^{\prime i} \Phi_{a}^{\prime} d \Omega_{a}^{\prime}=c n_{a} g^{i 0}, \quad W_{a}=\frac{1}{n_{a} m_{a} c^{2}} \int\left(p_{a}^{\prime 0}\right)^{2} \Phi_{a}^{\prime} d \Omega_{a}^{\prime}-1
$$

where prime means that all quantities under the integral are defined in the rest frame of the given plasma component, $d \Omega_{a}=d\left(\varepsilon_{a} / c\right) d^{3} p_{a}$ is the relativistic invariant, and $g^{i j}$ is the ordinary fundamental tensor in the noncurved space

$$
g^{i j}=g_{i j}=\left(\begin{array}{cc}
1 & 0 \\
0 & -\delta^{\alpha \beta}
\end{array}\right)
$$


In the nonrelativistic limit one can obtain for an isotropic plasma that $W_{a} \rightarrow \frac{3}{2} T_{a} /\left(m_{a} c^{2}\right)$, where $T_{a}$ is the temperature of the given plasma component in its proper frame. However, in relativistic case the problem of temperature definition is complicated (Javakhishvili and Tsintsadze 1973). It will be much more reasonable to consider $W_{a}$ as one of the macroscopic parameters, describing together with parameters $n_{a}$ and $\boldsymbol{u}_{a}$ the plasma hydrodynamical motion. The transition to the laboratory frame may be made by means of the Lorentz transformation for $p_{a}^{i}: p_{a}^{i}=L_{a}^{i j} p_{a j}$, where the Lorentz transformation matrix has the form (Landau and Lifshitz 1967)

$$
L_{a}^{i j}=\left(\begin{array}{cc}
\gamma_{a} & \gamma_{a} u_{a} / c \\
-\gamma_{a} u_{a} / c & -s_{a}^{\alpha \beta}
\end{array}\right)
$$

In eq. (7) $s^{\alpha \beta}$ is obtained in 3 -space as

$$
s_{a}^{\alpha \beta}=\delta^{\alpha \beta}+\left(\gamma_{a}-1\right) \frac{u^{\alpha} u^{\beta}}{u_{a}^{2}}, \quad \alpha=1,2,3
$$

Multiplying eq.(4) by 1 and $p_{a}^{i}$ and integrating over $d \Omega_{a}$, using equations (5) and (7) the continuity and energy-momentum equations may be obtained

$$
\frac{\partial J_{a}^{i}}{\partial x_{a}^{i}}=0, \quad \frac{\partial T_{a}^{i j}}{\partial x_{a}^{j}}=\frac{e_{a}}{c} F^{i j} J_{a j}, \quad T_{a}^{i j}=L_{a}^{m i} L_{a}^{n j} \pi_{a m n}
$$

where $J_{a}^{i}=L_{a}^{i j} I_{a j} ; J_{a}^{i}=\left(c \gamma_{a} n_{a}, n_{a} \gamma_{a} u_{a}\right)$ is the current 4-vector, and $T_{a}^{i j}$ is the energy-momentum tensor. The symmetrical tensor $\pi_{a}^{i j}$ is defined as

$$
\pi_{a}^{i j}=\int p^{\prime i} p^{\prime j} \Phi_{a}^{\prime} d \Omega_{a}^{\prime}
$$

Obviously $\pi_{a}^{00}=m_{a} c^{2} n_{a}\left(W_{a}+1\right)$.

The system (8) is not closed, because eq.(9) introduces some new undefined macroscopic values $\pi_{a}^{0 \alpha}$ and $\pi_{c}^{o: \beta}$. To close the system one should connect $\pi_{a}^{0 \alpha}$ and $\pi_{a}^{\alpha \beta}$ with the macroscopic parameters $n_{a}, \boldsymbol{u}_{a}, W_{a}$ and its derivatives. It should be done in the case when all quantities characterizing the plasma are slowly changing at distances of the order of the mean free path. It is shown below that this problem can be solved in case when the collisionless plasma is strongly magnetized, which leads to the anisotropy of thermal parameters describing plasma.

As is clear from (8), it is necessary to redefine $\pi_{a}^{0 \alpha}$ and $\pi_{a}^{\alpha \beta}$. It can be obtained $e . g$. by the construction of the additional transport equation for $\pi_{a}^{i j}$. This leads to the necessity of considering the equations for the third-order moments of the kinetic equation. For this purpose eq.(4) should be multiplied by $P_{a}^{i} P_{a}^{j}$ and integrated over $d \Omega_{a}$. This leads to

$$
\begin{aligned}
& \frac{\partial M_{a}^{i j k}}{\partial x_{a}^{k}}=\frac{e_{a}}{c}\left(F^{i m} T_{a m}^{j}-T_{a}^{i m} F_{m}^{j}\right), \\
& \text { where } \quad M_{a}^{i j k}=L^{e i} L^{m j} L^{n k} N_{a e m n}
\end{aligned}
$$

and $N_{a}^{i j k}$ is defined as

$$
N_{a}^{i j k}=\int p_{a}^{\prime i} p_{a}^{\prime j} p_{a}^{\prime k} \Phi_{a}^{\prime} d \Omega_{a}^{\prime}
$$

The $N_{a}^{i j k}$ and $\pi_{a}^{i j}$ tensors can be defined as

$$
\begin{gathered}
\pi_{a}^{i j}=\left(\begin{array}{cc}
m_{a} c^{2} n_{a}\left(W_{a}+1\right), & \boldsymbol{q}_{a} / c \\
\boldsymbol{q}_{a} / c, & \pi_{a}^{\alpha \beta}
\end{array}\right), \quad N_{a}^{i j 0}=m_{a} c\left(\begin{array}{cc}
m_{a} c^{2} n_{a} V_{a}, & 2 \boldsymbol{q}_{a} / c \\
2 \boldsymbol{q}_{a} / c, & \mu_{a}^{\alpha \beta}
\end{array}\right) \\
N_{a}^{\alpha \beta \gamma}=m_{a} \eta_{a}^{\alpha \beta \gamma}
\end{gathered}
$$

where it is convenient to define $n_{a}, W_{a}, V_{a}, \pi_{a}^{\alpha \beta}, \mu_{a}^{\alpha \beta}, \boldsymbol{q}_{a}, \boldsymbol{g}_{a}$ and $\eta_{a}^{\alpha \beta \gamma}$ using three dimensional distribution functions $f_{a}\left(\boldsymbol{r}_{a}, \boldsymbol{p}_{a}, t\right)$ in the following way (Javakhishvili and Tsintsadze 1973)

$$
n_{a}=\int f_{a}^{\prime} d \boldsymbol{p}_{a}^{\prime}, \quad W_{a}=\frac{1}{m_{a} c^{2} n_{a}} \int \varepsilon_{a}^{\prime} f_{a}^{\prime} d \boldsymbol{p}^{\prime}-1, \quad V_{a}=\frac{1}{m_{a}^{2} c^{4} n_{a}} \int \varepsilon_{a}^{\prime 2} f_{a}^{\prime} d \boldsymbol{p}_{a}^{\prime},
$$




$$
\begin{gathered}
\pi_{a}^{\alpha \beta}=c^{2} \int \frac{p_{a}^{\prime \alpha} p_{a}^{\prime \beta}}{\varepsilon_{a}^{\prime}} f_{a}^{\prime} d \boldsymbol{p}_{a}^{\prime}, \quad \mu_{a}^{\alpha \beta}=\frac{1}{m_{a}} \int p_{a}^{\prime \alpha} p_{a}^{\prime \beta} f_{a}^{\prime} d \boldsymbol{p}_{a}^{\prime}, \quad \boldsymbol{q}_{a}=c^{2} \int \boldsymbol{p}_{a}^{\prime} f_{a}^{\prime} d \boldsymbol{p}_{a}^{\prime} \\
\boldsymbol{g}_{a}=\frac{1}{2 m_{a}} \int \varepsilon_{a}^{\prime} \boldsymbol{p}_{a}^{\prime} f_{a}^{\prime} d \boldsymbol{p}_{a}^{\prime}, \quad c^{2} \int \frac{\boldsymbol{p}_{a}^{\prime}}{\varepsilon_{a}^{\prime}} f_{a}^{\prime} d \boldsymbol{p}_{a}^{\prime}=0, \quad \eta_{a}^{\alpha \beta \gamma}=\frac{c^{2}}{m_{a}} \int \frac{p_{a}^{\prime \alpha} p_{a}^{\prime \beta} p_{a}^{\prime \gamma}}{\varepsilon_{a}^{\prime}} f_{a}^{\prime} d \boldsymbol{p}^{\prime}
\end{gathered}
$$

Here all integrals are taken in the plasma rest frame; $\pi_{a}^{\alpha \beta}$ is the viscous stress tensor and $q_{a}$ is the heat flux density (Javakhishvili and Tsintsadze 1973). It can be seen that in the nonrelativistic limit $\left(\varepsilon^{\prime}=m c^{2}\right) \quad \mu_{a}^{\alpha \beta} \rightarrow \pi_{a}^{\alpha \beta}$ and the vector $g_{a}$ reduces to the $\boldsymbol{q}_{a}$. Therefore the tensor $\mu_{a}^{\alpha \beta}$ can be called the modified stress tensor and $g$ is the modified heat flux vector. Note that $S p\left(\mu_{a}^{\alpha \beta}\right)=\mu_{a}^{\alpha \alpha}=n_{a} m_{a} c^{2}\left(V_{a}-1\right)$. To close the set of equations one should e.g. neglect the third order moment $\eta_{a}^{\alpha \beta \gamma}$.

It is obvious that the kinetic equation for the function $f_{a}$ should be solved. This equation in the relativistic theory has the form (Belyaev and Budker 1956)

$$
\frac{\partial f_{a}}{\partial t}+\frac{c^{2}}{\varepsilon_{a}} \boldsymbol{p}_{a} \frac{\partial f_{a}}{\partial \boldsymbol{r}_{a}}+e_{a}\left\{\boldsymbol{E}+\frac{c}{\varepsilon_{a}}\left[\boldsymbol{p}_{a} \boldsymbol{B}\right]\right\} \frac{\partial f_{a}}{\partial \boldsymbol{p}_{a}}=0
$$

where $f_{a}$ is the relativistic invariant (Belyaev and Budker 1956) $d p_{a} / \varepsilon_{a}$. One should transform eq.(15) to the plasma rest frame. If $R_{a} / L \ll 1$, where $R_{a}$ is the Larmor radius of the particles, and $L$ is the characteristic spatial scale of the problem, one can look for the solution of eq.(15) in a form of power series over a small parameter $R_{a} / L$. Consequently the kinetic equation for $f_{a}^{\prime}$ reduces to the infinite set of equations

$$
\begin{gathered}
\left\{\boldsymbol{E}^{\prime}+\frac{c}{\varepsilon_{a}^{\prime}}\left[\boldsymbol{p}_{a}^{\prime} \boldsymbol{B}^{\prime}\right]\right\} \frac{\partial f_{a}^{\prime(0)}}{\partial \boldsymbol{p}_{a}^{\prime}}=0 \\
\frac{\partial f_{a}^{\prime(0)}}{\partial t}+\frac{c^{2}}{\varepsilon^{\prime}} \boldsymbol{p}_{a}^{\prime} \frac{\partial f_{a}^{\prime(0)}}{\partial \boldsymbol{r}_{a}}+e_{a}\left\{\boldsymbol{E}^{\prime}+\frac{c^{2}}{\varepsilon_{a}^{\prime}}\left[\boldsymbol{p}_{a}^{\prime} \boldsymbol{B}^{\prime}\right]\right\} \frac{\partial f_{a}^{(1)}}{\partial \boldsymbol{p}_{a}^{\prime}}=0
\end{gathered}
$$

If one limits by finding only the functions $f_{a}^{(0)}$, then the solution can be obtained easily by assuming $\boldsymbol{E}^{\prime}=0$.

$$
f_{a}^{\prime(0)}=f_{a}^{\prime(0)}\left(t, r_{a}, p_{a \perp}^{\prime 2}, p_{a}^{\prime} \cdot B^{\prime}\right)
$$

where $p_{a \perp}^{\prime 2}$ is transverse with respect to the $B^{\prime}$ component of the particle momentum.

Using the distribution function eq.(17) it can be shown from eq.(14) that $q_{a}=g_{a}=0$ and

$$
\pi_{a}^{\alpha \beta}=\mathbf{P}_{a \|} b^{\prime \alpha} b^{\prime \beta}+\mathbf{P}_{a \perp}\left(\delta^{\alpha \beta}-b^{\prime \alpha} b^{\prime \beta}\right), \quad \mu_{a}^{\alpha \beta}=\mu_{a \|} b^{\prime \alpha} b^{\prime \beta}+\mu_{a \perp}\left(\delta^{\alpha \beta}-b^{\prime \alpha} b^{\prime \beta}\right)
$$

where $\mathbf{P}_{a \|}, \mu_{a \|}$ and $\mathbf{P}_{a \perp}, \mu_{a \perp}$ are the parallel and transverse components of the pressure and modified pressure, respectively, which are defined as

$$
\begin{aligned}
& \mathbf{P}_{a \|}=c^{2} \int \frac{p_{a \|}^{\prime 2}}{\varepsilon_{a}^{\prime}} f_{a}^{\prime(0)} d^{3} p_{a}^{\prime}, \quad \mathbf{P}_{a \perp}=c^{2} \int \frac{p_{a \perp}^{\prime 2}}{2 \varepsilon_{a}^{\prime}} f_{a}^{\prime(0)} d^{3} p_{a}^{\prime} \\
& \mu_{a \|}=\frac{1}{m_{a}} \int p_{a \|}^{\prime 2} f_{a}^{\prime(0)} d^{3} p_{a}^{\prime}, \quad \mu_{a \perp}=\frac{1}{2 m_{a}} \int p_{a \perp}^{\prime 2} f_{a}^{\prime(0)} d^{3} p_{a}^{\prime}
\end{aligned}
$$

In the case considered $(q=0, g=0)$ and from eq.(18)) one can obtain

$$
\begin{gathered}
\pi_{a}^{i j}=\left[m_{a} c^{2} n_{a}\left(W_{a}+1\right)+\mathbf{P}_{a \perp}\right] g^{i 0} g^{j 0}-\mathbf{P}_{a \perp} g^{i j}+\left(\mathbf{P}_{a \|}-\mathbf{P}_{a \perp}\right) g^{i \alpha} g^{j \beta} b_{\alpha}^{\prime} b_{\beta}^{\prime}, \\
\frac{N^{i j 0}}{m_{a} c}=\left(m_{a} c^{2} n_{a} V_{a}+\mu_{a \perp}\right) g^{i 0} g^{j 0}-\mu_{a \perp} g i j+\left(\mu_{a \|}-\mu_{a \perp}\right) g^{i \alpha} g^{j \beta} b_{\alpha}^{\prime} b_{\beta}^{\prime}, \\
N_{a}^{\alpha \beta \gamma}=0
\end{gathered}
$$

Using the definitions (8), (11), and (20) and the Lorentz transformation matrix one can obtain

$$
\begin{gathered}
T_{a}^{i j}=\left[m_{a} c^{2} n_{a}\left(W_{a}+1\right)+\mathbf{P}_{a \perp}\right] u_{a}^{i} u_{a}^{j}-\mathbf{P}_{a \perp}\left(g^{i j}+\Lambda_{a}^{\alpha i} \Lambda_{a}^{\beta j} b_{\alpha} b_{\beta}\right)+\mathbf{P}_{a \|} \Lambda_{a}^{\alpha i} \Lambda_{a}^{\beta j} b_{\alpha} b_{\beta}, \\
\frac{M_{a}^{i j k}}{m_{a} c}=\left(m_{a} c^{2} n_{a} V_{a}+3 \mu_{a \perp}\right) u_{a}^{i} u_{a}^{j} u_{a}^{k}+
\end{gathered}
$$




$$
+\left(\mu_{a_{i} \mid}-\mu_{a \perp}\right)\left[u_{a}^{i} \Lambda_{a}^{\alpha j} \Lambda_{a}^{\beta k}+u_{a}^{j} \Lambda_{a}^{\alpha i} \Lambda_{a}^{\beta k}+u_{a}^{k} \Lambda_{a}^{\alpha i} \Lambda_{a}^{\beta j}\right]-\mu_{a \perp}\left[u_{a}^{i} g^{j k}+u_{a}^{j} g^{i k}+u_{a}^{k} g^{i j}\right]
$$

where $u_{a}^{i}\left(\gamma_{a}, \gamma_{a} u / c\right)$ is the 4-velocity of hydrodynamical motion and

$$
\Lambda_{a}^{i j} \equiv \frac{g^{i j}-u_{a}^{i} u_{a}^{j}}{\sqrt{1+\gamma_{a}^{2}\left(u_{a} \cdot b\right)^{2} / c^{2}}}
$$

Hence for hydrodynamical description of the relativistic collisionless plasma in a strong magnetic field, the set of macroscopic parameters $n_{a}, W_{a}, \mathbf{P}_{a \|}, \mathbf{P}_{a \perp}, \mu_{a \|}$ and $\mu_{a \perp}$ should be introduced, i.e. the number of parameters in the relativistic theory increases.

Let us now write the closed equation system for the set of macroscopic parameters, describing the relativistic strongly magnetized plasma. We have yet the continuity equation. From the second relation of eq.(8) at $i=\alpha$, using eq.(20) one can obtain the equation of motion. Multiplying this by $u_{a i}$ and summing up over $i$ one obtains the equation for $W_{a}$ (in fact it is a thermal balance equation)

$$
\begin{gathered}
n_{a} m_{a} c^{2} \frac{d}{d s_{a}}\left(W_{a}+\frac{\mathbf{P}_{a \perp}}{m_{a} c^{2} n_{a}}\right)-\frac{d \mathbf{P}_{a \perp}}{d s_{a}}-\left(\mathbf{P}_{a \perp}-\mathbf{P}_{a \|}\right) \Lambda_{a}^{\alpha i} \Lambda_{a}^{\beta j} b_{\alpha} b_{\beta} \frac{\partial u_{a i}}{\partial x_{a}^{j}}=0 \\
\text { where } \frac{d}{d s_{a}}=\left(u_{a}^{i} \frac{\partial}{\partial x_{a}^{i}}\right)=\frac{\gamma_{a}}{c}\left(\frac{\partial}{\partial t}+u_{a}^{\alpha} \frac{\partial}{\partial x_{a}^{\alpha}}\right)
\end{gathered}
$$

is the hydrodynamical derivative. In obtaining eq.(23) we used that $F^{i j} u_{a i} u_{a j} \equiv 0$. Multiplying eq.(10) by $u_{a j}$ one obtains the modified equation for energy-momentum

$$
u_{a j} \frac{\partial M_{a}^{i j k}}{\partial x_{a}^{k}}=\frac{e_{a}}{c}\left(F^{i m} T_{a m}^{j}-F_{m}^{j} T_{a}^{i j}\right) u_{a j}
$$

Excluding from eq.(10) the terms containing $d u_{a}^{i} / d s_{a}$ and $d V_{a} / d s_{a}$ leads to

$$
u_{a}^{i} u_{a}^{j} u_{a m} u_{a n} \frac{\partial M_{a}^{* m n k}}{\partial x_{a}^{k}}-u_{a}^{i} u_{a m} \frac{\partial M_{a}^{* j m k}}{\partial x_{a}^{k}}-u_{a}^{j} u_{a m} \frac{\partial M_{a}^{* i m k}}{\partial x_{a}^{k}}+\frac{\partial M_{a}^{* i j k}}{\partial x_{a}^{k}}=R_{a}^{i j}-R_{a}^{j m} u_{a}^{i} u_{a m}-R_{a}^{i m} u_{a}^{j} u_{a m}
$$

where $M_{a}^{* i j k} \equiv M_{a}^{i j k}-m_{a} c\left(m_{a} c^{2} n_{a} V_{a}+3 \mu_{a \perp} u_{a}^{i} u_{a}^{j} u_{a}^{k}\right)$, and $R_{a}^{i j}$ denotes the right-hand side of eq.(10). Let us introduce the projection operators

$$
D_{a i j}^{(l)}=\Lambda_{a \alpha i} \Lambda_{a \beta j} b^{\alpha} b^{\beta}, \quad D_{a i j}^{(t r)}=g_{i j}+D_{a i j}^{(l)}
$$

Multiplying eq.(25) by $D_{i j}^{(l)}$ and $D_{i j}^{(t r)}$ and summing over the indices $i, j$ one can obtain the equations for the macroscopic parameters $\mu_{\|}$and $\mu_{\perp}$

$$
\begin{aligned}
& \frac{d \mu_{a \|}}{d s_{a}}-\frac{\mu_{a \|}}{n_{a}} \frac{d n_{a}}{d s_{a}}-2 \mu_{a \|} \Lambda_{a}^{\alpha i} \Lambda_{a}^{\beta j} b_{\alpha} b_{\beta} \frac{\partial u_{a i}}{\partial x_{a}^{j}}=0 \\
& \frac{d \mu_{a \perp}}{d s_{a}}-\frac{2 \mu_{a \perp}}{n_{a}} \frac{d n_{a}}{d s_{a}}+\mu_{a \perp} \Lambda_{a}^{\alpha i} \Lambda_{a}^{\beta j} b_{\alpha} b_{\beta} \frac{\partial u_{a i}}{\partial x_{a}^{j}}=0
\end{aligned}
$$

Hence the equations (8) [the second part of eq.(8) at $i=0$ should be substituted with eq.(23)], eq.(24) [for $i=\alpha$ ] and eq.(27) then constitute a closed system of hydrodynamical equations for the relativistic collisionless plasma in the strong magnetic field. Eq.(24) connects the ordinary $\left(\mathbf{P}_{a \|}\right.$ and $\left.\mathbf{P}_{a \perp}\right)$ and the "modified" $\left(\mu_{a \|}, \mu_{a \perp}\right)$ macroscopic parameters of plasma. It is clear that this set of equations is the relativistic generalization of the Chew, Goldberger and Low theory for the nonrelativistic strongly magnetized plasma.

The relativistic case considered is caused by two reasons. First, the fact that the hydrodynamical velocity of plasma motion can be of the order of speed of light, and second, that the mean energy of plasma particles' thermal motion can be more than or of the order of the particle's rest energy. In the nonrelativistic limit $\left(u_{a} \ll c, T_{a} \ll m_{a} c^{2}\right)$ the system of hydrodynamical equations obtained reduces to a well known closed set of hydrodynamical equations (Baranov and Krasnobaev 1986). Indeed, in this case $W_{a} \rightarrow 0, V_{a} \rightarrow 1, \gamma_{a} \rightarrow 1, \Lambda^{\alpha i} \rightarrow g^{\alpha i}, \mu_{a \|} \rightarrow \mathbf{P}_{a \|}, \mu_{a \perp} \rightarrow \mathbf{P}_{a \perp}$, and the set of macroscopic parameters is reduced. In this limit eq.(27) coincides with the equations for the parallel and transverse pressure for the nonrelativistic case, and eq.(23) reduces to eq.(27) and becomes unnecessary. A detailed examination of these results will be published subsequently. 